\title{
ON A PROBLEM OF GRÜNBAUM
}

\author{
JACK G. CEDER
}

In [1, p. 249] Branko Grünbaum asked whether the set of all points which bisect three or more chords of a planar convex body was connected or even convex. In this note we show that this set is always polygonally connected but not necessarily convex.

Let $C$ be a given planar convex body. For each $\alpha \in[0, \pi)$, let $L_{\alpha}$, called a curve, denote the set of all points which are midpoints of chords of direction $\alpha$ which intersect the interior of $C$. Then each $L_{\alpha}$ is a subset of $C^{0}$ (the interior of $C$ ) and is the graph of a real-valued function which is the difference of two convex functions on some open interval. Let $\mathscr{L}$ denote the collection of all $L_{\alpha}$ for $\alpha \in[0, \pi)$.

First we establish the following lemma whose proof is clear and straightforward and hence, will be omitted.

LEMMA 1. (a) Each two curves in $\mathcal{L}$ intersect in either a closed line segment or in a single point.

(b) Two curves in $\mathfrak{L}$ intersect in a closed line segment if and only if there exists a pair of parallel line segments in the boundary of $C$.

(c) If there is a point $x$ common to $k$ curves in $\mathcal{L}$, then $x$ bisects at least $k$ chords of $C$.

(d) If $L_{\alpha} \cap L_{\beta}$ is not a single point, then each point of $L_{\alpha} \cap L_{\beta}$ bisects infinitely many chords.

If $L_{\alpha}, L_{\beta}$, and $L_{\gamma}$ are three noncurrent curves in $\mathscr{L}$ (i.e., $\left.L_{\alpha} \cap L_{\beta} \cap L_{\gamma}=\varnothing\right)$, then by the triangle determined by $L_{\alpha}, L_{\beta}$ and $L_{\gamma}$ we mean the nonempty closed set which is the union of the sets $L_{\alpha} \cap L_{\beta}, L_{\beta} \cap L_{\gamma}$ and $L_{\alpha} \cap L_{\gamma}$ with the closure of that component of $C-L_{\alpha}-L_{\beta}-L_{\gamma}$ whose closure lies entirely in $C^{0}$.

Lemma 2. Each point interior to the triangle determined by three nonconcurrent curves in $\mathcal{L}$ bisects at least three chords of $C$.

Proof. Let $L_{\alpha}$ and $L_{\beta}$ be in $\mathcal{L}$. And let $x$ be interior to one of the four components of $C^{0}-L_{\alpha}-L_{\beta}$. Then the directed distances from $x$ to $M_{\alpha} \cap L_{\alpha}$ and $M_{\beta} \cap L_{\beta}$ will be of different sign, where $M_{\alpha}$ and $M_{\beta}$ are the lines through $x$ in direction $\alpha$ and $\beta$, respectively. Since the directed distance is continuous, there will be a $\gamma$ "between" $\alpha$ and $\beta$ such that $x \in M_{\gamma} \cap L_{\gamma}$. Applying this argument to each of the three pairs of lines selected from those determining the given triangle, we easily obtain three distinct chords which $x$ bisects.

Received by the editors September 25, 1963. 
LEMMA 3. Let $T$ be any triangle formed by three nonconcurrent curves

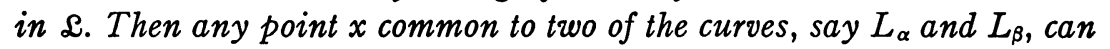
be joined to any point in $T^{0}$ by a polygonal arc lying in $T^{0} \cup\left(L_{\alpha} \cap L_{\beta}\right)$.

Proof. First of all, $T^{0}$ being a connected open subset of the plane is polygonally connected. Next let $z$ be the point in $L_{\alpha} \cap L_{\beta} \cap \bar{T}^{0}$. Then $z$ will be accessible to $T^{0}$ by a line segment (i.e., there exists a $w \in T^{0}$ such that $\left.(z, w] \subseteq T^{0}\right)$. For in case $L_{\alpha} \cap L_{\beta}$ is not a single point, the extension of the line segment $L_{\alpha} \cap L_{\beta}$ into $T^{0}$ is easily seen to yield such a line segment. And in case $L_{\alpha} \cap L_{\beta}=\{z\}$, there clearly exist distinct lines $M$ and $N$ through $z$ which are tangent to $C_{\alpha}$ and $C_{\beta}$, respectively (any curve in $\mathcal{L}$ being the graph of a function of bounded variation has left and right tangents everywhere). Then it easily follows that the extension into $T^{0}$ of any line "between" $M$ and $N$ and passing through $z$ yields such a line segment.

Now let $x \in L_{\alpha} \cap L_{\beta}$ and $y \in T^{0}$ and let $z$ and $w$ be as specified above. Then $[x, z] \cup[z, w] \cup P(w, y)$, where $P(w, y)$ is a polygonal arc joining $w$ to $y$ in $T^{0}$, will have a polygonal arc as a subset which joins $x$ to $y$ in $\left(L_{\alpha} \cap L_{\beta}\right) \cup T^{0}$.

THEOREM. The set of points which bisect three or more chords of a planar convex body is polygonally connected.

Proof. Let $x$ and $y$ be two such points. Then clearly there will exist two triangles, $S$ and $T$, whose interiors intersect such that $x$ (resp. $y$ ) belongs to two "sides" of $S$ (resp. $T$ ). Letting $w \in S^{0} \cap T^{0}$ we can, by applying Lemma 3 , obtain the appropriate polygonal arcs joining $w$ to $x$ and $y$, respectively. From these two arcs we can obtain a polygonal arc joining $x$ and $y$ which contains, by Lemmas 1 and 2, only points which bisect three or more chords.

As an example to show that the set of points, $B$, bisecting three or more chords may fail to be convex, take a regular pentagon $P$. It can be shown without difficulty that $B$ has the shape of a "five-pointed star." Specifically, let $v_{1}, \cdots, v_{5}$ denote (in order) the vertices of $P$; let $M_{i}$ be the chord joining $v_{i}$ to the midpoint of the opposite side; let $y_{i}$ be the point of intersection of $M_{i}$ and the chord $\left[v_{i-1}, v_{i+1}\right](\bmod 5)$; and let $w_{i}$ be the point of intersection of $M_{i}$ and the segment $\left[y_{i-2}, y_{i-3}\right](\bmod 5)$. Then it can be shown that $B$ is contained in the closed convex hull of $y_{1}, \cdots, y_{5}$. Moreover, $y_{i} \in \bar{B}$ and $w_{i} \notin \bar{B}$ for each $i$. Hence, $B$ is not convex.

\section{BIBLIOGRAPHY}

1. Branko Grünbaum, Measures of symmetry for convex sets, Convexity, Proc. Sympos. Pure Math. Vol. 7, pp. 233-270, Amer. Math. Soc., Providence, R. I., 1963.

University of California, Santa Barbara 